\title{
Separation characteristics and structure optimization of double spherical tangent double-field coupling demulsifier
}

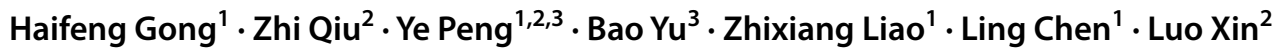

Received: 21 April 2021 / Accepted: 26 October 2021 / Published online: 7 November 2021

(c) The Author(s) 2021

\begin{abstract}
The demulsification and dewatering of the W/O emulsion are widely used in petrochemical industry, oilfield exploitation, and resource and environmental engineering. However, efficiently treating emulsion via traditional single methods. In this study, a new double-field coupling demulsification and dewatering device is proposed, where the conical structure of the device is double spherical tangential type. The numerical model for double-field coupling is established, especially, the population balance model (PBM) is used to simulate the coalescence and breakup of dispersed droplets under the double-field coupling action. And the effects of three conical structures on the internal flow and separation efficiency are analyzed. Results show that the conical structure has a significant effect on the coalescence of droplets, especially the double spherical tangential cone is more conducive for improving the coalescence ability of small droplets and improving the separation efficiency of the device. After optimization, the optimal $R$ value of the double spherical tangential coupling device is $300 \mathrm{~mm}$, and the separation efficiency can be up to $96.32 \%$, which is $6.13 \%$ higher than the separation efficiency of the straight double-cone coupling device.
\end{abstract}

Keywords Double spherical cone $\cdot$ Demulsification $\cdot$ Separation characteristics $\cdot$ Double-field coupling $\cdot$ Coalescence

\section{List of symbols}

$\mathrm{c}_{1} \quad$ A constant of order unity

$\mathrm{P} \quad$ The internal pressure of the fluid, $\mathrm{Pa}$

D Nominal diameter, $\mathrm{mm}$

$\mathrm{r}_{1}, \mathrm{R} \quad$ Spherical radius of small cone and big cone, $\mathrm{mm}$

$\mathrm{D}_{\mathrm{o}} \quad$ Overflow port diameter, $\mathrm{m}$

$\mathrm{v}_{\mathrm{w}}, \mathrm{v}_{\mathrm{o}} \quad$ Oil and water velocity, $\mathrm{m} \cdot \mathrm{s}-1$

$\mathrm{D}_{\mathrm{s}} \quad$ Cyclone chamber diameter, $\mathrm{mm}$

$\mathrm{v}_{\mathrm{r}} \quad$ Two phases through the relative velocity, m.s -1

$\mathrm{D}_{\mathrm{t}} \quad$ Entrance diameter, $\mathrm{mm}$

$\alpha \quad$ Big cone angle, ${ }^{\circ}$

$\mathrm{D}_{\mathrm{u}} \quad$ Underflow port diameter, $\mathrm{mm}$

Zhi Qiu

qzchongqing@163.com

1 Engineering Research Centre for Waste Oil Recovery Technology and Equipment of Ministry of Education, Chongqing 400067, China

2 Chongqing Key Laboratory of Manufacturing Equipment Mechanism Design and Control, Chongqing Technology and Business University, Chongqing 400067, China

3 State Key Laboratory of Heavy Oil Processing, China University of Petroleum (East China), Qingdao 266580, China $\alpha_{\mathrm{o}}, \alpha_{\mathrm{w}} \quad$ Oil and water volume fraction, $\%$

$\mathrm{E} \quad$ Electric field amplitude, $\mathrm{kv} \cdot \mathrm{m}-1$

$\beta \quad$ Small cone angle, ${ }^{\circ}$

$\mathrm{F}_{\mathrm{e}} \quad$ Electric field force, $\mathrm{N}$

$\varepsilon_{0} \quad$ Vacuum dielectric constant, F.m-1

I Unit tensor

$\varepsilon_{\mathrm{o}}, \varepsilon_{\mathrm{w}} \quad$ Oil and water phase dielectric constant, F.m-1

$\mathrm{K}_{\mathrm{i}}, \mathrm{K}_{\mathrm{j}} \quad$ Number densities, $\mathrm{kg} \cdot \mathrm{m}-3$

$\zeta \quad$ Arc angle of big cone, ${ }^{\circ}$

$\mathrm{L}_{\mathrm{i}}, \mathrm{L}_{\mathrm{j}} \quad$ Two colliding droplet diameters, mm

$\mu_{\mathrm{o}}, \mu_{\mathrm{w}} \quad$ Oil and water phase viscosity, mpa $\mathrm{s}$

$\mathrm{L}_{\mathrm{s}} \quad$ Overflow pipe length, $\mathrm{mm}$

$\rho \quad$ Density of emulsion, $\mathrm{kg} \cdot \mathrm{m}-3$

$\mathrm{L}_{\mathrm{t}} \quad$ Cone length, $\mathrm{mm}$

$\rho_{\mathrm{o}}, \rho_{\mathrm{w}} \quad$ Oil and water density, $\mathrm{kg} \cdot \mathrm{m}-3$

$\mathrm{L}_{\mathrm{u}} \quad$ Underflow pipe length, mm

$\sigma_{\mathrm{o}}, \sigma_{\mathrm{w}}$ Oil and water phase conductivity, s.m-1

$\mathrm{L}_{1}, \mathrm{~L}_{2} \quad$ Small cone and big cone length, $\mathrm{mm}$

w Emulsion velocity, $\mathrm{m} \cdot \mathrm{s}-1$ 


\section{Introduction}

Water in oil (W/O) emulsion generally exists in petrochemical industry, oilfield exploitation, development of heavy oil reservoirs, and resource and environmental engineering fields (Liu et al. 2020; Lv et al. 2020). The W/O emulsion is a colloidal dispersion of oil and water, where the water phase is dispersed in the oil phase (Ghasemi et al. 2020). At present, the global energy demand is continuously increasing (Almeida-Trasvina et al. 2021). The recycling of emulsion is particularly important, and demulsification and dewatering are the most critical step in the process of resource recycling (Praporgescu and Mihăilescu 2011; Lam et al. 2016; Raya et al. 2020). Physical, chemical, and biological methods are used for emulsion treatment (Kang et al. 2018; Long et al. 2013; Gu et al. 2015). The most common methods are physical methods, including electric field, rotary displacement, gravity settlement, and vacuum heating methods (Qian et al. 2017; Mhatre et al. 2015; Li et al. 2015; Qi et al. 2018).

In general, it is difficult to achieve demulsification and dewatering of emulsion efficiently by using a single processing method (Liu and Wang 2016; Jung et al.2019; Hamza et al. 2020; Qiu et al. 2020; Gong et al. 2018). The demulsification and dewatering can be well achieved by using various processing methods or by coupling an integrated unit operation, which is also the key direction of future development (Eow and Ghadiri 2002; Vashahi et al. 2019; Xu et al. 2020; Motin and Bénard 2017). The electric field method refers to the stretching deformation of dispersed droplets in oil under the action of electric field, which aggravates the collision of droplets, promotes the coalescence of droplets in emulsion, and increases the size of droplets (Mozaffari et al. 2021; Bijesh et al. 2018). However, this method cannot achieve the effect of rapid and efficient separation (Wang et al. 2016). Centrifugation is generally applied in the separation process that involves the different densities of oil and water in a separator to effectively and quickly achieve the oil-water separation. The oil phase with a small density flows out from the center area of the device to the overflow orifice; the water phase with a high density moves along the wall of the device to the underflow orifice. Therefore, the coupling of electric and swirl centrifugal fields can achieve a better effect of droplet size enlargement and rapid and effective separation. They complement each other's advantages, thereby improving the demulsification and dewatering efficiency of the emulsion. For example, Bailes and Watson (1992) investigated the demulsification of water in crude oil emulsion by a high-voltage pulsed DC electric field and proposed a continuous rotating electrostatic demulsifier. Therefore, emulsion can be effectively dewatered and purified under the combined action of electric field and centrifugal fields. Ghadiri and Eow (2003) studied the demulsification and dewatering treatments of emulsion under the combined action of pulsed direct current electric and centrifugal fields. They proposed a centrifugal electric coalescence separator to make the droplets coalesce quickly under the action of double-field coupling and improve the separation efficiency. Adamski et al. (2018) investigated the agglomeration and separation system and the process of emulsion under the action of centrifugal force and electric field to make the droplets in the emulsion coalesce under the action of a double field. Then, they used the separator for effective separation.

Studies have focused on the influence of inlet velocity, voltage amplitude, and inlet structure on the separation efficiency of the device, ignoring the important influence of the cone structure on the separation efficiency of the cyclone device. For example, Zhang et al. (2020) investigated the influence of different voltages and inlet flow rates on the separation efficiency of emulsion under the coupling effect of electric and swirl centrifugal fields and obtained the optimal voltage amplitude and inlet velocity. Noik and Trapy (2004) studied the influence of device inlet structure type on emulsion separation efficiency under the action of electric and swirl centrifugal field and found that cone-shaped inlet type was more conducive to emulsion swirl separation. Al-Kayiem et al. (2019) studied the effects of different inlet forms on the separation performance of the device and found that the flow field of the dual inlet was more stable than that of the single inlet, which was more conducive to improving the separation efficiency of the device. Al-Kayiem et al. (2020) investigated the influence of the vane's deflection angle in an oil/water axial inlet hydrocyclone separator on the separation efficiency. The study found that the separation efficiency obtained by using the $45^{\circ}$ swirl generator was higher than other angles. However, the influence of the conical structure on the separation performance of the device has not been studied.

Therefore, in this paper, the electric and centrifugal fields coupling device is taken as the research object and research and analysis are conducted from the cone type of the device. A straight double-cone structure (Fig. 1) does not have a smooth connection and has a gradient shape, which causes the vibration and the instability of the flow field, affecting the separation efficiency. This study proposes a new type of double-field coupling device where the double spherical tangent cone is applied (Fig. 2). The double spherical tangent structure (Fig. 3) achieves a smooth connection in the double-cone section. Consequently, the flow field becomes more stable and can effectively eliminate the adverse factors. In this paper, under the different cone conditions, numerical calculations were conducted on the emulsion using computational fluid dynamics (CFD) and the population balance model (PBM) to obtain the coupling device's oil-water 


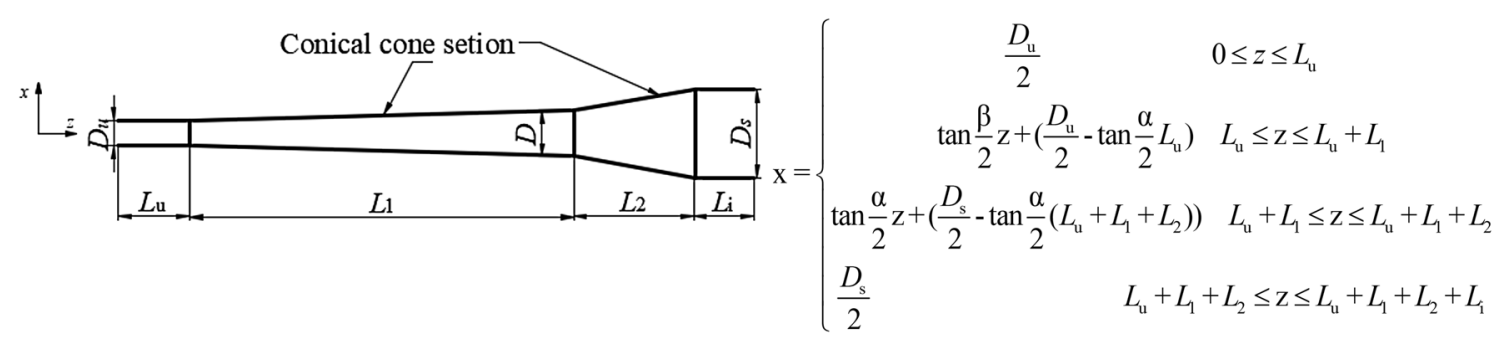

Fig.1 Straight double-cone structure

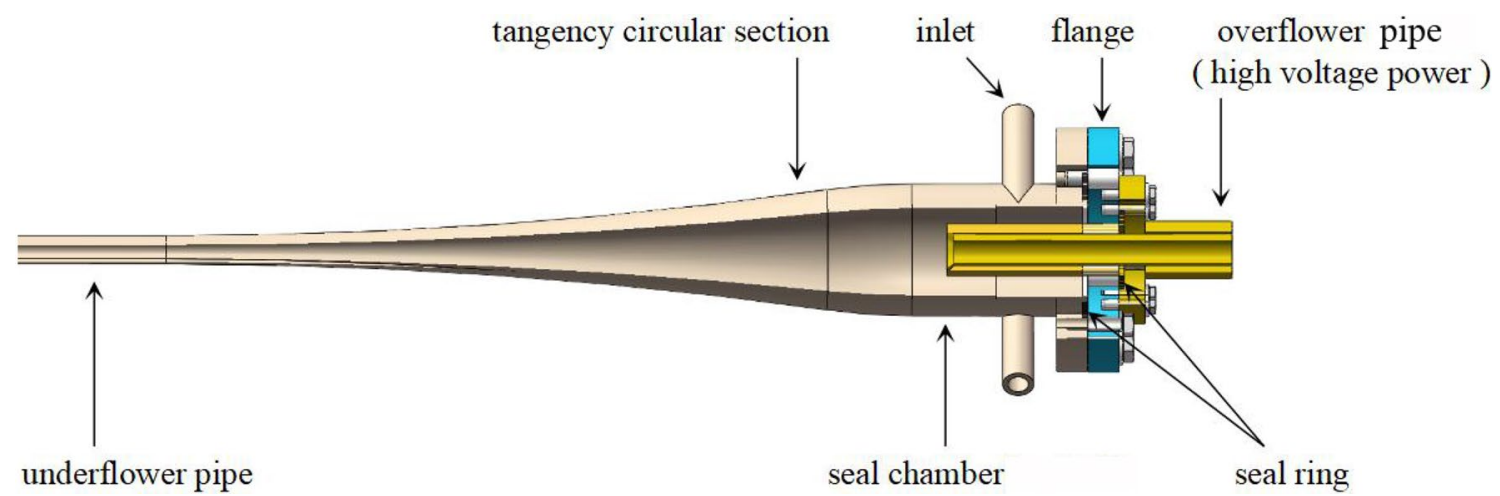

Fig. 2 Schematic diagram of double-field coupling dewatering device

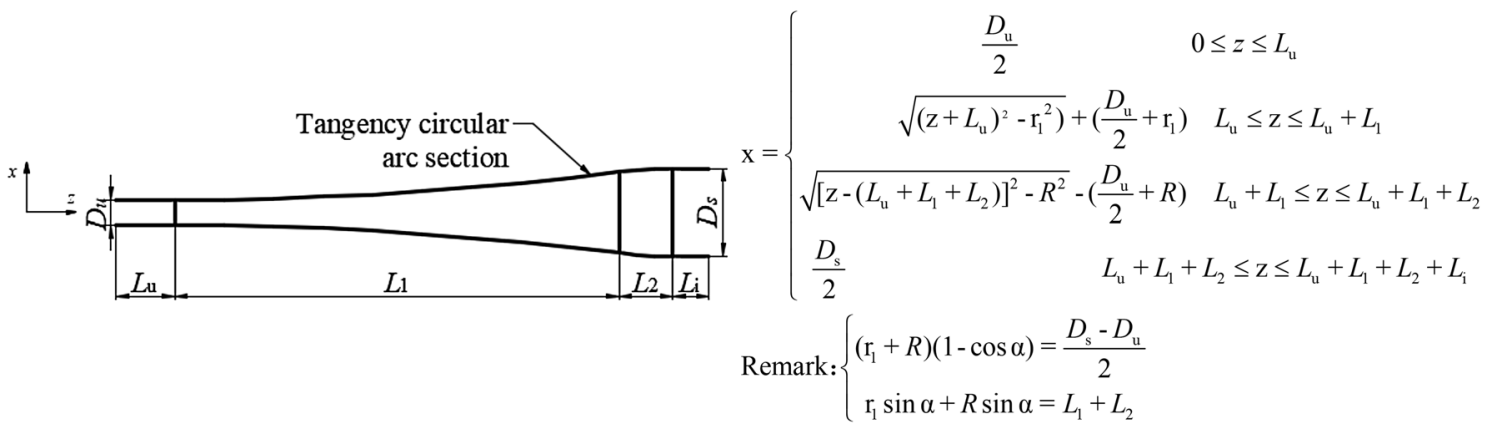

Fig. 3 Double spherical tangent cone structure

separation performance. The cone structure of the coupling device with two spherical tangents is optimized. In addition, this present study can explore a new structure with higher separation performance and provide insightful references for the design of a new double-field coupling device.

\section{Model}

\section{Double-field coupling device}

Figure $4 \mathrm{a}$ shows the structure of the double-sphere tangent-type double-field coupling separation device, which is mainly composed of a double tangential inlet, a swirl chamber, a double-sphere tangent section, and a straight 
Fig. 4 Structural of the different cone coupling separation device: a the double spherical tangent cone; $\mathbf{b}$ the straight double-cone

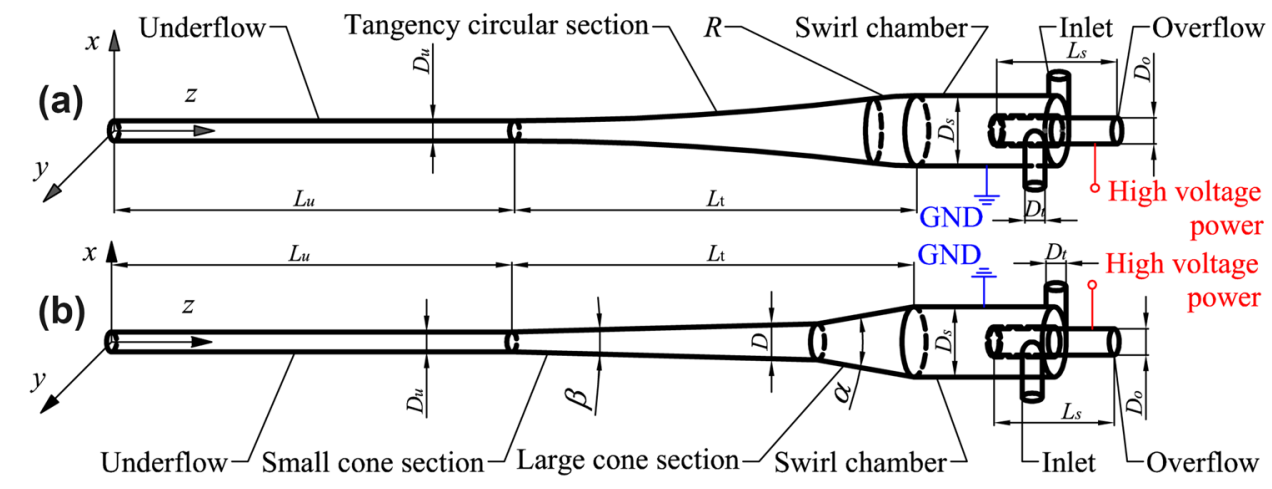

Table 1 Structural parameters of the coupling separation device

\begin{tabular}{llllllllll}
\hline$D_{\mathrm{u}} / \mathrm{mm}$ & $L_{\mathrm{u}} / \mathrm{mm}$ & $L_{\mathrm{t}} / \mathrm{mm}$ & $D_{\mathrm{s}} / \mathrm{mm}$ & $L_{\mathrm{s}} / \mathrm{mm}$ & $D_{\mathrm{o}} / \mathrm{mm}$ & $D_{\mathrm{t}} / \mathrm{mm}$ & $D / \mathrm{mm}$ & $\alpha /^{\circ}$ & $\beta / /^{\circ}$ \\
\hline 10 & 400 & 430 & 70 & 70 & 18 & 12 & 30 & 20 & 3 \\
\hline
\end{tabular}

pipe section near an underflow outlet. An electric field is embedded in the swirl chamber (i.e., the outer wall of the swirl chamber is grounded, and the outer wall of the overflow pipe is connected to the positive pole of the power supply). Under the action of the electric field, small emulsion droplets stretch and deform in the emulsion, intensify the collision of droplets, promote the coalescence of emulsion droplets, increase the droplet size, and then achieve rapid and effective separation under the action of centrifugal force. Figure $4 \mathrm{~b}$ illustrates the schematic of the traditional straight double-cone structure, and Table 1 lists the structural parameters of the coupling separation device.

\section{Governing equations}

The continuity equation and momentum conservation equation are expressed as follows (Cernecky.and Plandorova 2013):

$\frac{\partial \rho}{\partial t}=-\nabla \cdot \sum_{i=\mathrm{w}, \mathrm{o}}\left(\rho_{i} a_{i} v_{i}\right)$

$$
F_{e}=\nabla \cdot\left[\left(1-\frac{I}{2}\right) \varepsilon_{0} \varepsilon E E\right]
$$

\section{Coalescence and breakup equations}

Considering the aggregation and fragmentation of droplets under the action of electric field force, the Luo model is adopted for the analysis in this study (Zhang et al. 2020). Then, the volume formation rate of particles generated via secondary collision is expressed as follow:

$\Omega\left(L_{i}, L_{j}\right)=f\left(L_{i}, L_{j}\right) p\left(L_{i}, L_{j}\right)$

where $f\left(L_{i}, L_{j}\right)$ is the collision frequency, and $p\left(L_{i}, L_{j}\right)$ is the coalescence probability.

The collision frequency is determined on the basis of the relative velocity, particle size, and bulk density of the two droplets, so $f\left(L_{i}, L_{j}\right)$ is defined as:

$f\left(L_{i}, L_{j}\right)=\frac{\pi}{4}\left(L_{i}, L_{j}\right)^{2} K_{i} K_{j} v_{i j}$

\section{Electric field equation}

In this study, a user-defined function (UDF) is used to couple the electric field governing equation with the flow field governing equation (Zhang et al. 2020). The electric field force of the droplet in the two-field coupling element is described as follows:
When the relative collision velocity of the centerline of the two droplets is lower than the critical value, they will not coalescence (Howarth 1964). In addition, the contact time after oil-water collision determines the coalescence of droplets. When $t_{d}>t_{c}$, the two droplets cannot coalesce. In turbulence, the contact time of colliding droplets is (Motin 2015): 
$t_{c}=\left(L_{i}+L_{j}\right)^{2 / 3} \varepsilon^{-1 / 3}$

$t_{d}=\frac{5.8 \mu_{\mathrm{w}}}{F}\left(\frac{L_{i} L_{j}}{L_{i}+L_{j}}\right)^{3 / 2}\left(h_{i}^{1 / 2}-h_{f}^{1 / 2}\right)$

where $F$ is the force between the droplets; $\mu_{w}$ is the viscosity of liquid droplets; $h_{i}$ is the initial thickness of liquid film; $h_{f}$ is the final thickness of liquid film rupture. In this study, the probability of droplet coalescence is described as follows:

$p\left(L_{i}, L_{j}\right)=\operatorname{esp}\left(-t_{d} / t_{c}\right)$

$p\left(L_{i}, L_{j}\right)=\operatorname{esp}\left\{-\frac{0.76 c_{1}\left[\left[1+\left(L_{i} / L_{j}\right)^{2}\right]\left[1+\left(L_{i} / L_{j}\right)^{3}\right]\right]^{1 / 2}}{\left[1+\left(L_{i} / L_{j}\right)\right]^{3}}\left(\rho L_{i} v_{\mathrm{ij}}^{2} / \sigma\right)^{1 / 2}\right\}$

The electric field accelerates the cracking of oil film and effectively promotes the droplet coalescence. However, if the electric field strength is too high, the droplet may be broken, thereby causing electric dispersion. Atten (1993) gave the expression of the critical electric field, which leads to droplets to break:

$E_{c}=0.64\left(\frac{\sigma}{\varepsilon_{\mathrm{o}} \varepsilon_{\mathrm{v}} d}\right)^{1 / 2}$

According to the formula, when the electric field intensity is $10^{6} \mathrm{~V} / \mathrm{m}$ (approximately $14 \mathrm{kV}$ ), the droplet with a diameter larger than $1.16 \mathrm{~mm}$ will be broken. Admittedly, the droplet size is less than $1.16 \mathrm{~mm}$ in the experiment. Therefore, the electric field dissipation is ignored in this study. where $\tau_{\mathrm{c}}$ is the critical shear stress for droplet breaking; $\sigma$ is the tension coefficient; $L_{\mathrm{w}}$ is the droplet diameters.

\section{Volume fraction equation of dispersed phase}

According to Rusche (2003) and (Oliveira and Issa 2003), the volume fraction equation of the dispersed phase is expressed as follows Cheng et al. (2018):

$\frac{\partial \alpha_{\mathrm{w}}}{\partial \mathrm{t}}+\nabla \cdot\left(v \alpha_{w}\right)+\nabla\left(v_{\mathrm{r}} \alpha_{w}-v_{\mathrm{r}} \alpha_{w}^{2}\right)=0$
Volume fraction equation of continuous phase Li (2020)

$\frac{\partial\left(\alpha_{\mathrm{o}} \rho_{\mathrm{o}}\right)}{\partial \mathrm{t}}+\frac{\partial\left(\alpha_{\mathrm{o}} \rho_{\mathrm{o}} v\right)}{\partial x}+\frac{\partial\left(\alpha_{\mathrm{o}} \rho_{\mathrm{o}} v_{\mathrm{r}}\right)}{\partial x}=0$

\section{Solution method of population balance equations (PBE)}

The PBE could predict droplet size distribution well (SaeedMozaffari et al. 2019) and is solved by discrete method and moments method. In this study, to obtain the droplet size distribution directly, we applied the discrete method developed by Ramkrishna. In ANSYS Fluent, the discrete PBE is expressed as (Ansys Incorporation 2015 and Gong et al. 2020):

$\frac{\partial N_{i}}{\partial t}+\nabla\left(u_{i} N_{i}\right)=\sum_{k=1}^{N} \sum_{j=1}^{N} a_{k j} N_{k} N_{j} x_{k j} \varsigma_{k j}+\sum_{j=i+1}^{N} g_{v j} \varphi_{i, k} N_{j} \beta_{i j}-\sum_{j=1}^{N} a_{i j} N_{i} N_{j}-g_{v i} N_{i}$

Based on the Hagesaether method (Hagesaether et al. 2002), the breaking formula of the discrete method is described as follows: where $a_{i j}$ is the special coalescence frequency between two droplets with size of $x_{\mathrm{i}}$ and $x_{\mathrm{j}} ; N_{i}$ and $N_{j}$ are the volumetric number concentration of droplets with volume $V_{i}$ and $V_{j} ; g_{\mathrm{v}}$

$B_{k}(i)=\sum_{z=i+1, i \neq n}^{n} \Omega_{k}\left(v_{z}, v_{i}\right)+\sum_{z=i, i \neq n}^{i} x_{i+1}, z \Omega_{k}\left(v_{i+1}, v_{z}\right)+\sum_{z=1, i \neq 1}^{i-1}\left(1-x_{i, z}\right) g\left(v_{i+1} \Omega_{k}\left(v_{i}, v_{z}\right)\right)$

where $\Omega_{k}\left(v_{z}, v_{i}\right)=n_{z} g\left(v_{z}\right) \beta\left(v_{z}, v_{i}\right)$.

In this study, we found that when the shear force of the droplet is lower than the pressure difference between the inside and the outside of the interface film, the model cannot predict the droplet breaking process. The critical conditions are:

$\tau_{\mathrm{c}}=4 \sigma / L_{\mathrm{w}}$ is the integral droplet volume fraction at underflow; $\varsigma_{k j}$ is the criteria function to judge whether the droplet formed by the combination of droplet $\mathrm{k}$ and $\mathrm{j}$ reaches a certain droplet size interval $(i)$, and is defined as: 
$\varsigma_{k j}=\left\{\begin{array}{l}1 \text { for } V_{i}<V_{a g}<V_{i+1}, \text { where } i \leq N-1 \\ 0 \text { otherwise }\end{array}\right.$

$V_{a g}$ is the particle volume resulting from the aggregation of particles $k$ and $j$, and is defined as:

$V_{a g}=x_{k j} V_{i}+\left(1-x_{k j}\right) V_{i+1}$

where $x_{k j}$ is the distribution function, and is defined as:

$x_{k j}=\frac{V_{a g}-V_{i+1}}{V_{i}-V_{i+1}}$

Also, in order to preserve the properties of breakage droplets, introduce the function $\varphi_{i, k}$ to measure the fractional distribution of breakage droplets to the nearby characteristic sizes, and is defined as:

$\varphi_{i, k}=\int_{V_{i}}^{V_{i+1}} \frac{V_{i+1}-V}{V_{i+1}-V_{i}} \omega\left(V, V_{k}\right) \mathrm{d} V+\int_{V_{i-1}}^{V_{i}} \frac{V-V_{i-1}}{V_{i}-V_{i-1}} \omega\left(V, V_{k}\right) \mathrm{d} V$

\section{Grid independence analysis}

The device model is divided into three different mesh numbers: $134615,388,405$, and 711,769 . The static pressure and tangential velocity of different mesh numbers at the section $\mathrm{z}=700 \mathrm{~mm}$ are shown in Fig. 5. The optimal number of grids is found by comparing the distribution of tangential velocity and static pressure. In Fig. 5, the simulation results are almost the same when the number of grids is 388405 and 711,769 . Considering the time consumption and calculation results, the appropriate number of meshing is 388405 .

\section{Boundary conditions}

The physical parameters of the emulsion used in the experiment are shown in Table 2.

The normal velocity of inlet is $v=10\left(\mathrm{~m} \cdot \mathrm{s}^{-1}\right)$, the volume fraction of oil in the emulsion is $\alpha=90 \%$, the turbulence intensity at the inlet of the coupling separation device is $T=5 \%$, the split ratio of the upper outlet of the coupling device is $O_{\mathrm{FRW}}=0.9$, and the split ratio of the lower outlet of the coupling separation device $U_{\mathrm{FRW}}=0.1$. For the electric field region of the device, the outer wall of the overflow pipe is added with $11 \mathrm{kV}$ high voltage, and the other boundary surfaces are 0 ; the inlet type is set as the velocity-inlet, and the overflow and underflow orifices type are set to outflow. The near-wall surface is treated with the standard wall function, and the wall surface is set as the nonslip boundary condition.

The Reynolds stress model (RSM) is chosen in the simulation because it makes the $\mathrm{N}-\mathrm{S}$ equation to be closed and reduces the Reynolds pressure in the equation and the dissipation rate. In RSM, rapid changes in streamline bending, vortex, swirl, and tension are considered more strictly, and the model has a more accurate prediction ability for a complex flow. In simulation analysis, a pressure-based solver is adopted, and the "SIMPLEC" algorithm is adopted to realize the coupling effect of velocity and pressure (Chu et al. 2017; Vakamalla et al. 2014). In spatial discretization, gradient, pressure, momentum, volume fraction, turbulent kinetic energy, turbulent dissipation rate, and Reynolds stress are analyzed by using the least squares element and PRESTO!, QUICK (Chu et al. 2017; Vakamalla et al. 2014). Because the least square element has the characteristics of high precision and high efficiency on an irregular unstructured
Fig. 5 Static pressure and tangential velocity at the section $\mathrm{z}=750 \mathrm{~mm}$ under different numbers of grids (a)

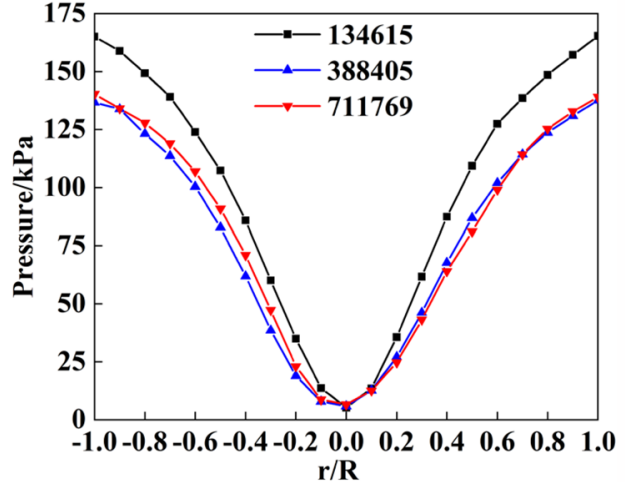

(b)

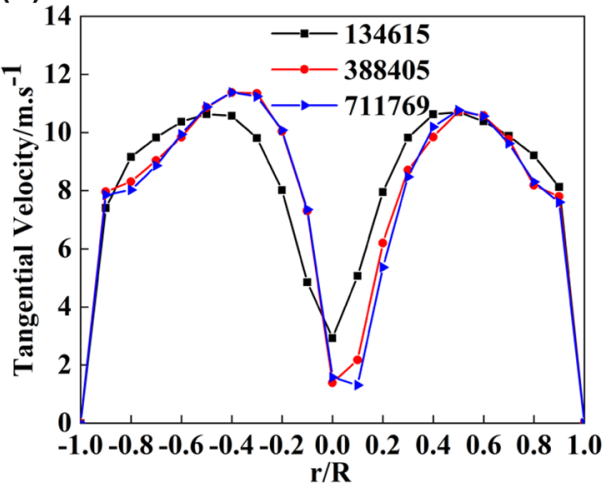

Table 2 physical parameters of emulsion

\begin{tabular}{llllllll}
\hline$\rho_{\mathrm{o}}\left(\mathrm{kg} \cdot \mathrm{m}^{-3}\right)$ & $\rho_{\mathrm{w}}\left(\mathrm{kg} \cdot \mathrm{m}^{-3}\right)$ & $\varepsilon_{\mathrm{o}}\left(\mathrm{F} \cdot \mathrm{m}^{-1}\right)$ & $\varepsilon_{\mathrm{w}}\left(\mathrm{F} \cdot \mathrm{m}^{-1}\right)$ & $\sigma_{\mathrm{o}}\left(\mathrm{s} \cdot \mathrm{m}^{-1}\right)$ & $\sigma_{\mathrm{w}}\left(\mathrm{s} \cdot \mathrm{m}^{-1}\right)$ & $\mu_{0}(\mathrm{mPa} \cdot \mathrm{s})$ & $\mu_{\mathrm{w}}(\mathrm{mPa} \cdot \mathrm{s})$ \\
\hline 863 & 998.2 & 2.65 & 81 & 0.0098 & 0.02 & 16.8 & 1.003 \\
\hline
\end{tabular}


grid (Ansys Incorporation 2013). In the swirling device, the three-dimensional flow pressure has a higher precision discretization ability under the action of PRESTO! (Ansys Incorporation 2013).

\section{Experimental methods}

\section{Experimental materials and devices}

The emulsion used in the experiments is prepared by injecting water into No. 25 transformer oil and mixing it at a ratio of 1:9. The physical parameters of the emulsion are shown in Table 2 and all parameter values are measured under the condition of $20^{\circ}$. In the preparation, span- 80 emulsifier was added at a concentration of $5 \mathrm{~g} / \mathrm{L}$ to maintain the stability of the emulsion, and an electric stirrer (MGD699) was set for stirring for $15 \mathrm{~min}$. After the mixture is allowed to stand for $24 \mathrm{~h}$, no free water precipitation is present, so it meets the experimental requirements. The prepared emulsion is placed in the storage tank for standby. The separation device used in this experiment is shown in Fig. 6. The main components are as follows: single pump, coupling device with straight double-cone structure, high-voltage power supply, pressure gauge, overflow tank, underflow tank, tee, and valves. The purpose of the tee is to ensure that the two-way inlet flow velocity is the same and that it is cut in to enter the device so that the separation efficiency is higher under the effect of the centrifugal field. During the operation of the device, the emulsion enters the device, and the oil-water separation is carried out under the coupling effect of the highvoltage electric field and the swirl centrifugal field. The high-concentration oil is discharged from the overflow port into the overflow tank, and the high-concentration water is discharged from the underflow port of the device into the underflow tank. After a certain period, the sampling valve of the overflow tank and the bottom flow tank is opened to extract a small amount of liquid. The moisture content of the sample droplets is measured with a petroleum hygrometer (SYD-2122C), and the separation efficiency of the emulsion is calculated by using the formula (20). During the experiment, the pressure change at any time should be explored to avoid the low pressure affecting the separation efficiency and high-pressure, causing safety accidents.

$E_{\mathrm{dw}}=1-\frac{f_{\mathrm{wo}}}{f_{\mathrm{w}}}$

Here $f_{\text {wo }}$ is the water volume fraction discharged from overflow orifice, $f_{\mathrm{w}}$ is water volume fraction of prepared emulsion.

\section{Experimental procedure}

Before running the device, complete the following steps: (1) place the equipment stably and ensure that there is power supply around the coupling device; (2) check the tightness and smoothness inside the device; (3) ensure that the line is connected, the part embedded in the high-voltage electric field is insulated, and the power supply voltage gear is set to zero.

When the power is turned on and the device is running, the following steps are operated: first, turn on the single screw pump. Then, after the device works stably, turn on the high-voltage pulse power supply and set various parameters

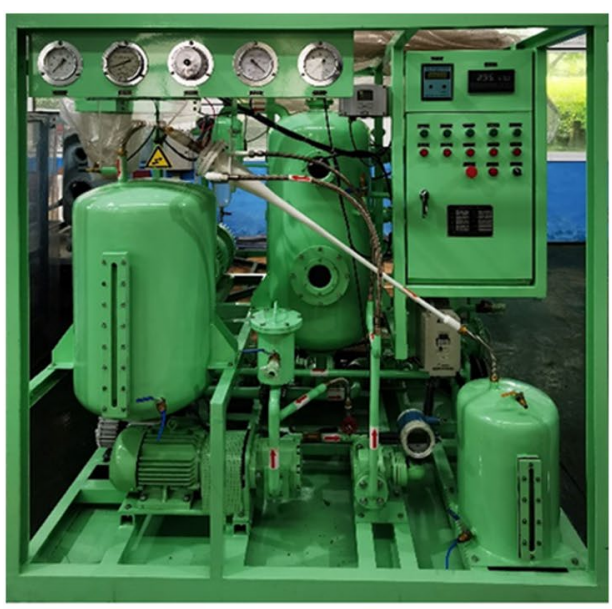

(a)

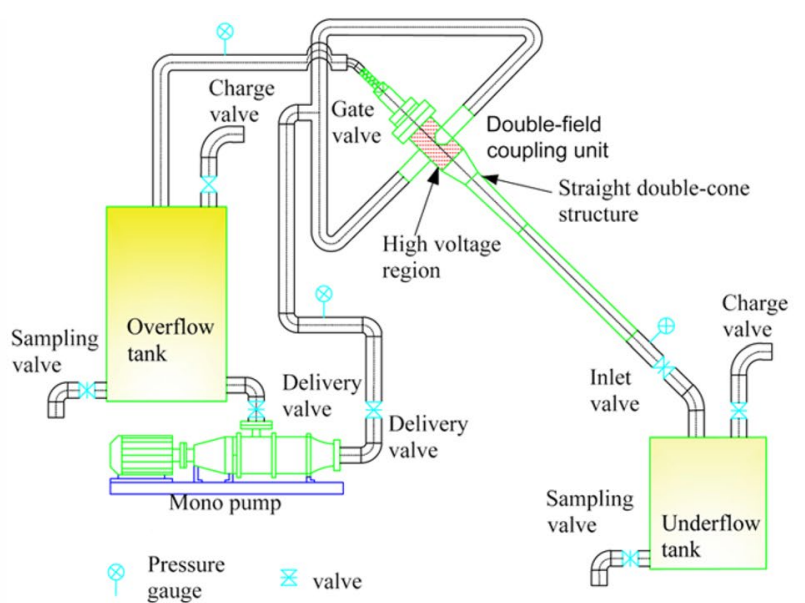

(b)

Fig. 6 a experimental of double-field coupling demulsification and dewatering and $\mathbf{b}$ schematic diagram of double-field coupling demulsification and dewatering 
Fig. 7 a the microscopy images of the emulsions before and after separation; $\mathbf{b}$ experimental and numerical results of the separation device under different voltage conditions (a)

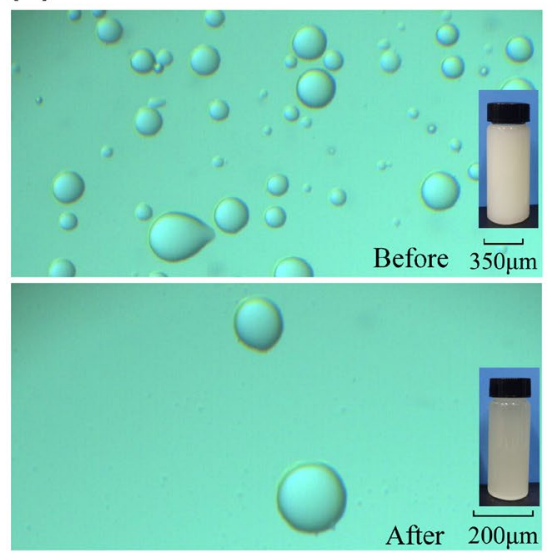

(b)

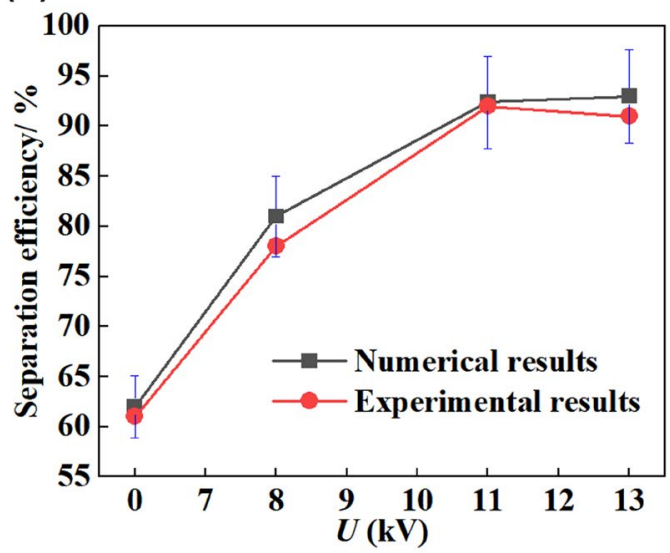

for the experiment. Finally, after the experiment, disconnect the high-voltage pulse power supply, and close each valve and the main power supply after the emulsion is drained.

\section{Results and discussion}

\section{Model validation}

The separation efficiency obtained via the experimental method and numerical simulation is compared to verify the rationality of the numerical model (Fig. 7). The experimental data in Fig. 7 are the average of five experiments. In Fig. 7 , the experimental results are in agreement with the numerical results. However, the numerical results are slightly larger than the experimental results because numerical simulation is carried out under relatively ideal conditions, ignoring the change in the temperature $e$ of the material in the experiment, the influence of the friction of the mechanical operation on the emulsion, and other factors. However, the difference between the numerical and experimental results is acceptable, indicating that the model is feasible. Therefore, the model is suitable for the double-field coupling separation device.

\section{Structure optimization}

The structure of the swirl device can be determined only by confirming a sphere radius $R$ because the distance value of the double-cone section along the $\mathrm{z}$-axis of the swirl device is certain, and the double spheres, the sphere, and the straight pipe section are tangent. The range of the sphere radius $R$ is $100-3000 \mathrm{~mm}$. The corresponding dewatering and deoiling rate are shown in Fig. 8. At a voltage of $U=11 \mathrm{kV}$ and an inlet velocity of $v=10 \mathrm{~m} / \mathrm{s}$, only single factor $R$ changes. In Fig. 8, the optimal sphere radius $R$ is $300 \mathrm{~mm}$.

\section{Separation characteristics of the coupling device}

\section{Static pressure distribution}

Static pressure distribution not only plays an important role in the flow of emulsion in the swirling device but also
Fig. 8 Distribution of deoiling and dewatering rates at different $R$

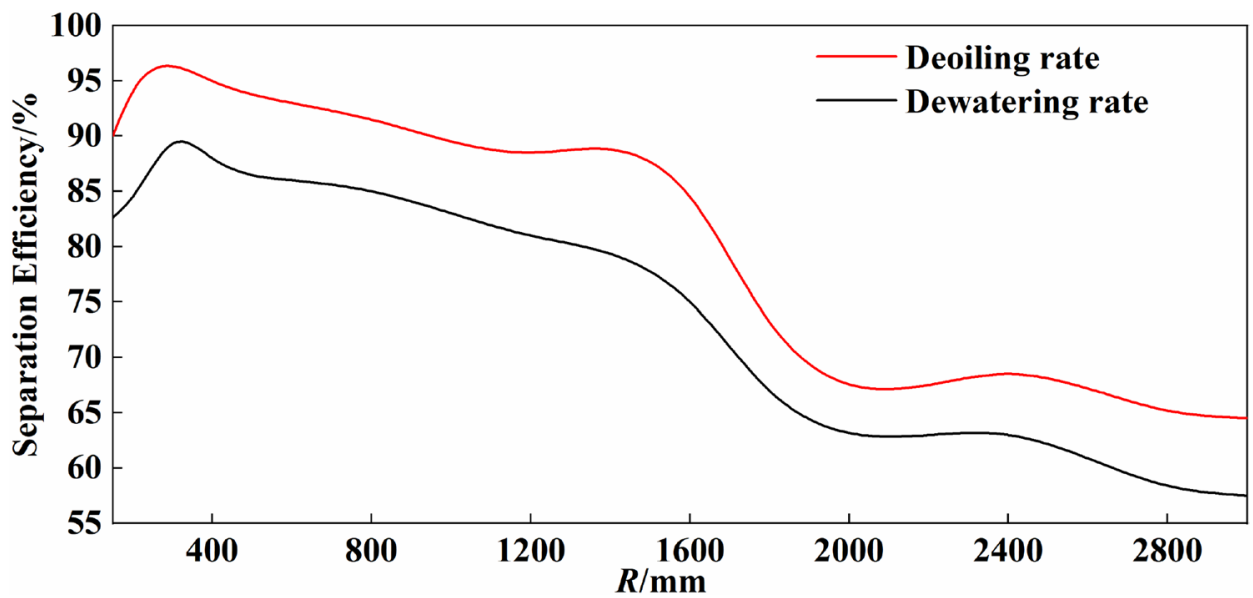




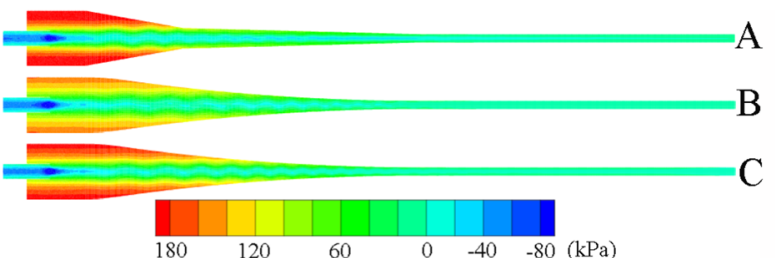

(a)
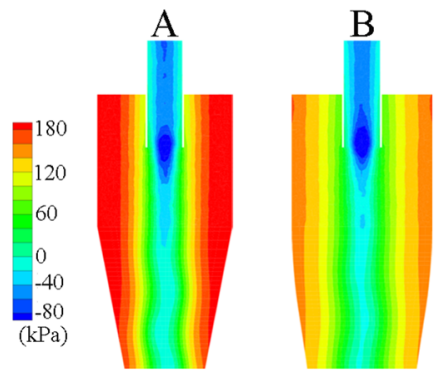

(b)
$\mathrm{B}$

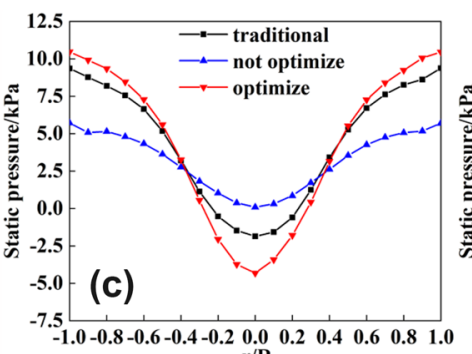

$\mathbf{r} / \mathbf{R}$

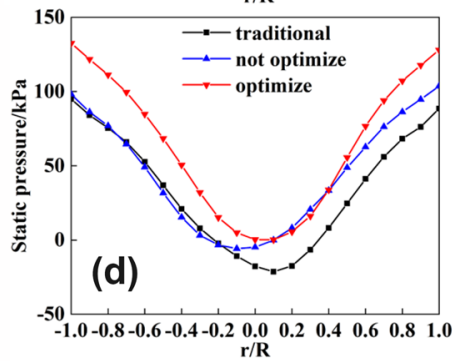

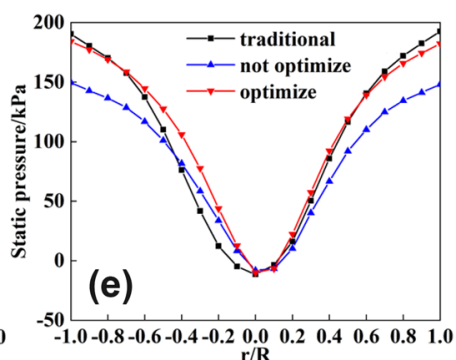

$\mathbf{r} / \mathbf{R}$

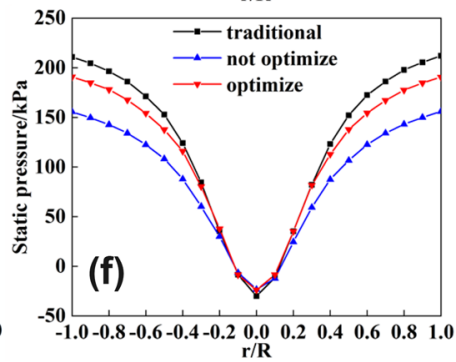

Fig. 9 a Contours of static pressure distribution at $\mathrm{x}=0 \mathrm{~mm}$ section (A: straight double-cone structure, B: not optimized double spherical tangent cone structure, and C: optimized double spherical tan-

affects the separation performance and energy loss of the swirling device (Tian et al. 2020). The static contours of pressure distribution at $\mathrm{x}=0 \mathrm{~mm}$ section under different cone structure is shown in Fig. 9. According to the figure, static pressure distribution is centrosymmetric because the device is double tangential inlet. The static pressure decreases with the decrease of $\mathrm{z}$-axis value. The reason is that the fluid rotates in the device and overcomes the pressure loss caused by the friction of fluid viscosity and fluid steering. The static pressure is positively correlated with the radius. As the radius decreases, the static pressure decreases, which is consistent with the distribution trend of the static pressure inside the coupling device (Chen et al. 2015). In the overflow pipe region, the negative pressure of the double spherical tangent coupling device is smaller than that of the straight double-cone coupling device, which is more conducive to the flow with higher oil content flowing out of the overflow port. The radial distribution of static pressure at different sections is shown in Fig. 9c-f. It can be seen from the figure that the negative pressure value of the optimized double spherical tangent coupling device near the underflow port $(\mathrm{z}=130 \mathrm{~mm})$ and the axis is large, which is $132.15 \%$ higher than that of the straight double-cone coupling device, which is conducive to the discharge of liquid with higher water content from the underflow port. Near the overflow port $(z=840 \mathrm{~mm})$, the negative pressure value of the optimized double spherical tangent coupling device in the axial region is small, which is $20.58 \%$ lower than that of the straight double-cone coupling device, which is conducive to the flow with higher oil content discharges from the overflow port and improve the gent cone structure); $\mathbf{b}$ at $\mathrm{x}=0$ and $750 \mathrm{~mm}<\mathrm{z}<935 \mathrm{~mm}$. (c)-(f) static pressure distribution of at different sections: $\mathbf{c} z=130 \mathrm{~mm}, \mathbf{d}$ $\mathrm{z}=650 \mathrm{~mm}, \mathbf{e} \mathrm{z}=780 \mathrm{~mm}, \mathbf{f} \mathrm{z}=840 \mathrm{~mm}$

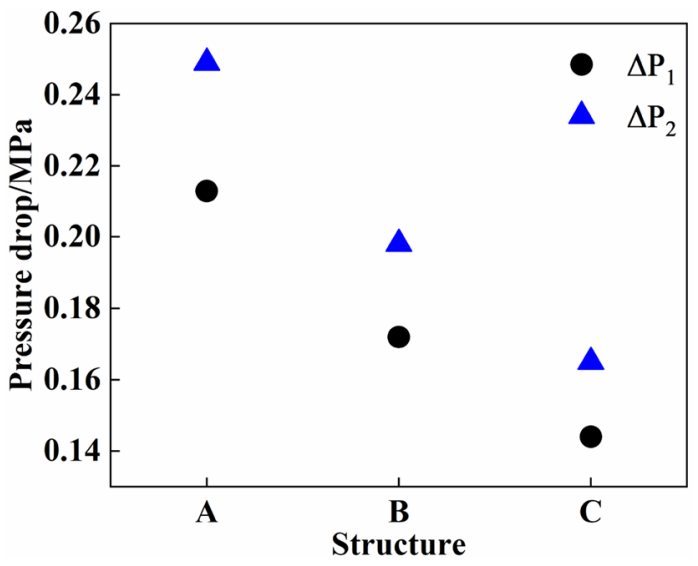

Fig. 10 Effect of different structures on pressure drop (A: straight double-cone structure, B: not optimized double spherical tangent cone structure, and C: optimized double spherical tangent cone structure)

separation efficiency. In the cone region, the static pressure value and pressure gradient of the optimized double spherical tangential coupling device are large, which indicates that the internal energy loss of the device is small and the energy consumption is low.

The pressure difference $\Delta p_{1}$ between the device inlet and the overflow port and the pressure difference $\Delta p_{2}$ between the feed port and the underflow port of the coupling device is shown in Fig. 10. $\Delta p_{2}$ is more important because it indicates the amount of the pressure loss of the liquid flowing through the coupling device. The pressure drop is defined as follows (Chen et al. 2015). 
$\Delta p_{1}=p_{i}-p_{y}$

$\Delta p_{2}=p_{i}-p_{u}$

In Fig. 10, $\Delta p_{2}$ of the three structures is greater than $\Delta p_{1}$, that is, the pressure drop ratio $\left(\Delta p_{2} / \Delta p_{1}\right)$ is greater than 1 , which is consistent with the research results of Chen et al. (2015). $\Delta p_{2}$ of the optimized double spherical tangent structure is reduced by $33.7 \%$ compared with $\Delta p_{1}$ of the straight double-cone structure because the optimized double spherical tangent structure reduces the emulsion flow resistance. A reasonable device structure should have as little pressure drop as possible, because the lower the pressure drop, the smaller the energy loss. In addition, the pressure is beneficial to drive the flow of the emulsion (Mozaffari et al. 2017), which is more conducive to the oil-water separation of emulsion in the swirl device. Therefore, the optimized structure is more conducive to the oil-water separation of emulsion.

(a)

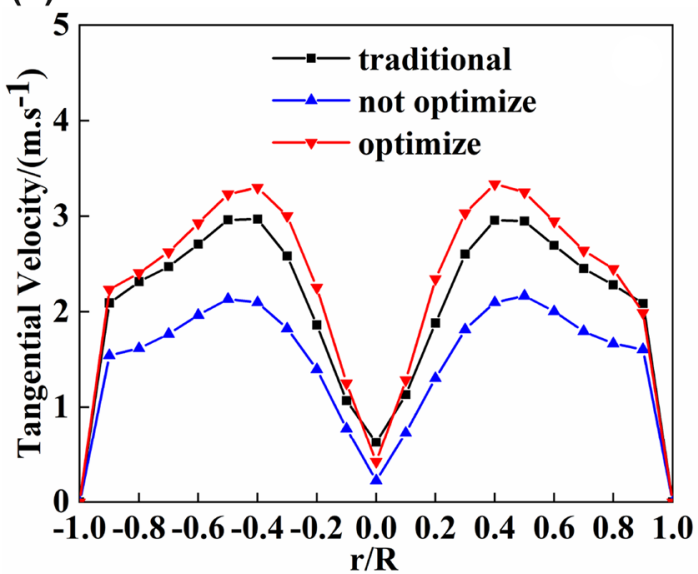

(c)

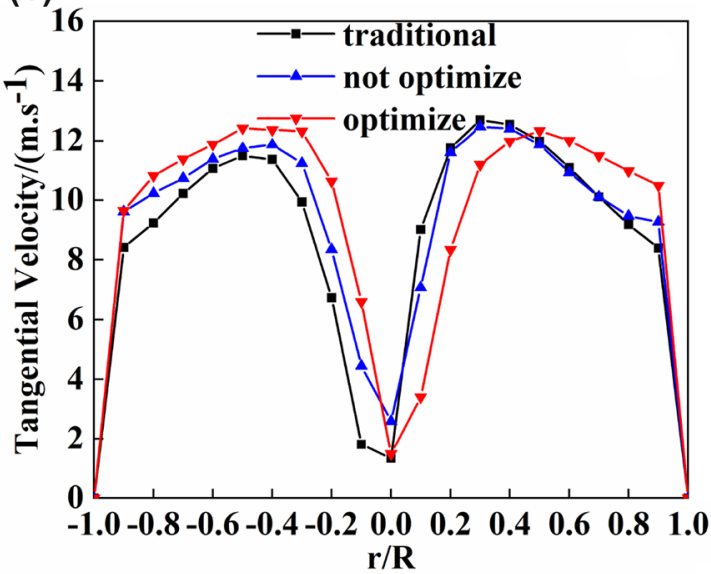

\section{Tangential velocity distribution}

Under the action of high-speed rotation in the separation device, different centrifugal forces are produced because of the density difference between oil and water, to achieve the effect of oil-water separation. The greater the tangential velocity in the same section is, the greater the centrifugal force on the droplets in the swirling device is, which is conducive to the droplet migration to the wall area of the swirling device, and more conducive to the swirling separation (Chen et al. 2015). However, a larger tangential velocity does not indicate a better outcome. If the tangential velocity is too large, droplets become deformed and broken by shear force, which aggravates the emulsification of oil and reduces the separation efficiency. Therefore, the tangential velocity is controlled within a certain range as much as possible. The radial distribution curve of tangential velocity on different sections is shown in Fig. 11. It shows with, with the increase of radius, the tangential velocity first increases and then decreases to zero. The tangential velocity of the three structures is small near the axis and wall. With the decrease

(b)

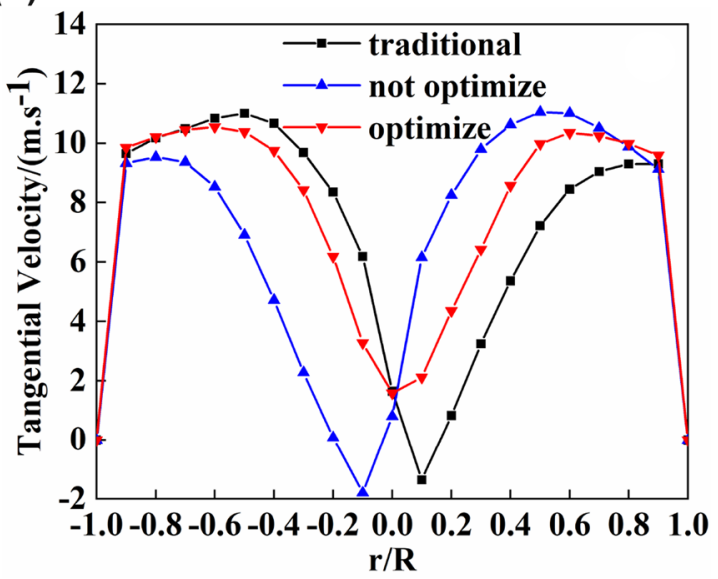

(d)

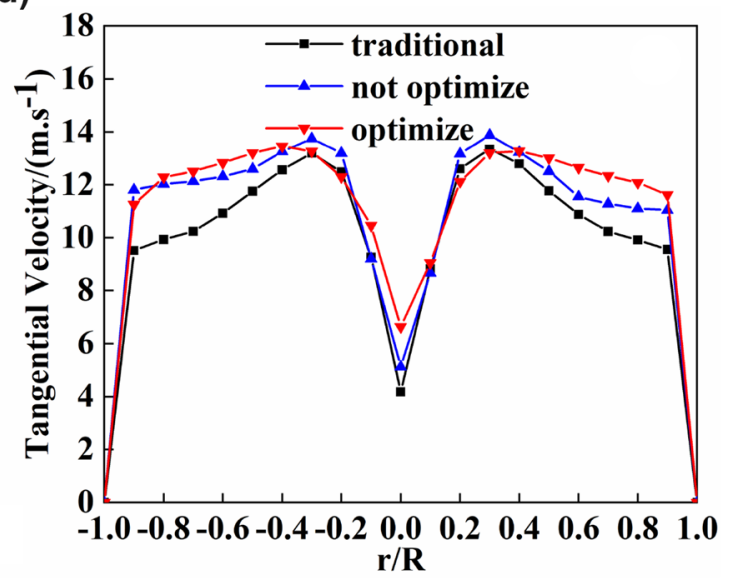

Fig. 11 Tangential velocity distribution of three structures at different sections $\mathbf{a} \mathrm{z}=130 \mathrm{~mm}, \mathbf{b} \mathrm{z}=650 \mathrm{~mm}, \mathbf{c} \mathrm{z}=780 \mathrm{~mm}, \mathbf{d ~ z}=840 \mathrm{~m}$ 
of $\mathrm{z}$ coordinate value, the tangential velocity decreases, and the surface along the $\mathrm{z}$ coordinate value decreases, which leads to energy loss. As can be seen from Fig. 11, near the underflow outlet $(\mathrm{z}=130 \mathrm{~mm}$ section), the tangential velocity of the optimized double spherical tangent coupling device is higher than that of the straight double-cone type and the non-optimized double spherical tangent coupling device, which indicates that the droplets are more likely to move toward the wall due to greater centrifugal force, facilitating the separation of oil and water. In the cone region, the tangential velocity distribution of the straight double-cone coupling device and the non-optimized double spherical tangent coupling device have obvious deviation, and the tangential velocity distribution of the optimized double spherical tangent coupling device is symmetrical, indicating that the internal flow of the structure is stable, which is conducive to oil-water separation. At $\mathrm{z}=780 \mathrm{~mm}$ section, the tangential velocity of the optimized double spherical tangent coupling device is larger in the range of $0.5<|\mathrm{r} / \mathrm{R}|<0.9$, which is $25.03 \%$ higher than that of the straight double-cone coupling device, indicating that the droplet is subjected to greater centrifugal force, which promotes the droplet to move toward the wall and is more conducive to the separation of oil and water. Near the overflow port $(\mathrm{z}=840 \mathrm{~mm}$ section), the tangential velocity of the optimized double spherical tangential coupling device is larger than that of the straight biconical coupling device, and the tangential velocity of the optimized double spherical tangent coupling device is $58.90 \%$ higher than that of the straight double-cone coupling device in the axial region, which reduces the water content in the axial region. It is favorable for the flowing liquid with high oil content to flow out from the overflow port and promote the oil-water separation.

\section{Oil volume fraction distribution}

The volume distribution of the oil volume fraction can reflect the movement of the $\mathrm{W} / \mathrm{O}$ emulsion during the separation in the coupling device. The oil volume fraction distributions of the three structures are shown in Fig. 12. In Fig. 12, the volume fraction of the oil volume fraction in the area near the inner wall of the swirl device decreases as the z-axis decreases and reaches the maximum value near the overflow port and the minimum value near the underflow port. The oil volume fraction decreases as the radius increases, and the oil phase is concentrated in the central area of the axis so that a stable oil core is formed in the central area. At this time, the maximum value is reached at the center of the axis, which is consistent with the oil phase distribution studied by Gong et al. (2020).

In Fig. 12, near the overflow port ( $\mathrm{z}=840 \mathrm{~mm}$ section), the high oil volume fraction, could promote the emulsion with high oil content to flow out from the overflow port, to achieve a high separation efficiency. It can be seen from the figure that at the cross section of $\mathrm{z}=840 \mathrm{~mm}$, the oil volume fraction of the optimized double spherical tangent coupling device in the axial region $(0<|r / R|<0.2)$ is $0.64 \%$ higher than that of the straight double-cone coupling device, indicating that the oil volume fraction of the optimized structure near the overflow port is large, which is more conducive to the discharge of the fluid with higher oil content from the overflow port and improves the separation efficiency of the device. Near the underflow port $(\mathrm{z}=130 \mathrm{~mm}$ section), the oil volume fraction of the non-optimized double spherical tangent coupling device is larger than that of the straight double-cone and optimized double spherical tangent coupling device. In the area of $0.5<|\mathrm{r} / \mathrm{R}|<1$, the oil volume fraction of the optimized double spherical tangential coupling device is $36.61 \%$ lower than that of the straight double-cone coupling device, indicating that the oil volume fraction of the liquid discharged from the underflow port by the optimized double spherical tangential coupling device is small and the separation effect of the device. At $\mathrm{z}=650 \mathrm{~mm}$ section, the oil volume fraction difference of the optimized double spherical tangent coupling device is 0.39 , which is $8.33 \%$ and $30 \%$ higher than that of the straight double-cone coupling device and the non-optimized double spherical tangent coupling device, which indicates that the optimized double spherical tangent coupling device is more conducive to oil-water separation and improves the separation efficiency.

\section{Droplet diameter distribution}

Droplet diameter plays a decisive role in the centrifugal force on the emulsion. The water in the emulsion is separated by the centrifugal force in the swirl device, and the droplet diameter is an important factor affecting the centrifugal force (Yang et al. 2012; Schütz et al. 2009). Figure 13 shows the droplet diameter distribution of the straight double-cone, the non-optimized double spherical tangent, and the optimized double spherical tangent coupling devices at $x=0 \mathrm{~mm} \mathrm{sec}$ tion. In Fig. 13a, small droplets collide and coalesce under the coupling effect of double fields, and the droplet diameter gradually increases along the $\mathrm{z}$-axis of the distance from the coupling device. In the axial region, effectively coalesce the droplets in the emulsion is difficult because of the small centrifugal force on the droplets, so the droplet diameter is small in the axial region of the coupling device. The double-cone section area is the main area of separation. In Fig. 13b, the droplet size of the straight double spherical tangent coupling device is the smallest, which is the least conducive to the swirling separation of droplet. The droplet size of the optimized double spherical tangent coupling device is the largest, indicating that the droplet is subject to great centrifugal force, and the oil-water separation is more sufficient, which is more conducive to improve the separation efficiency. In 

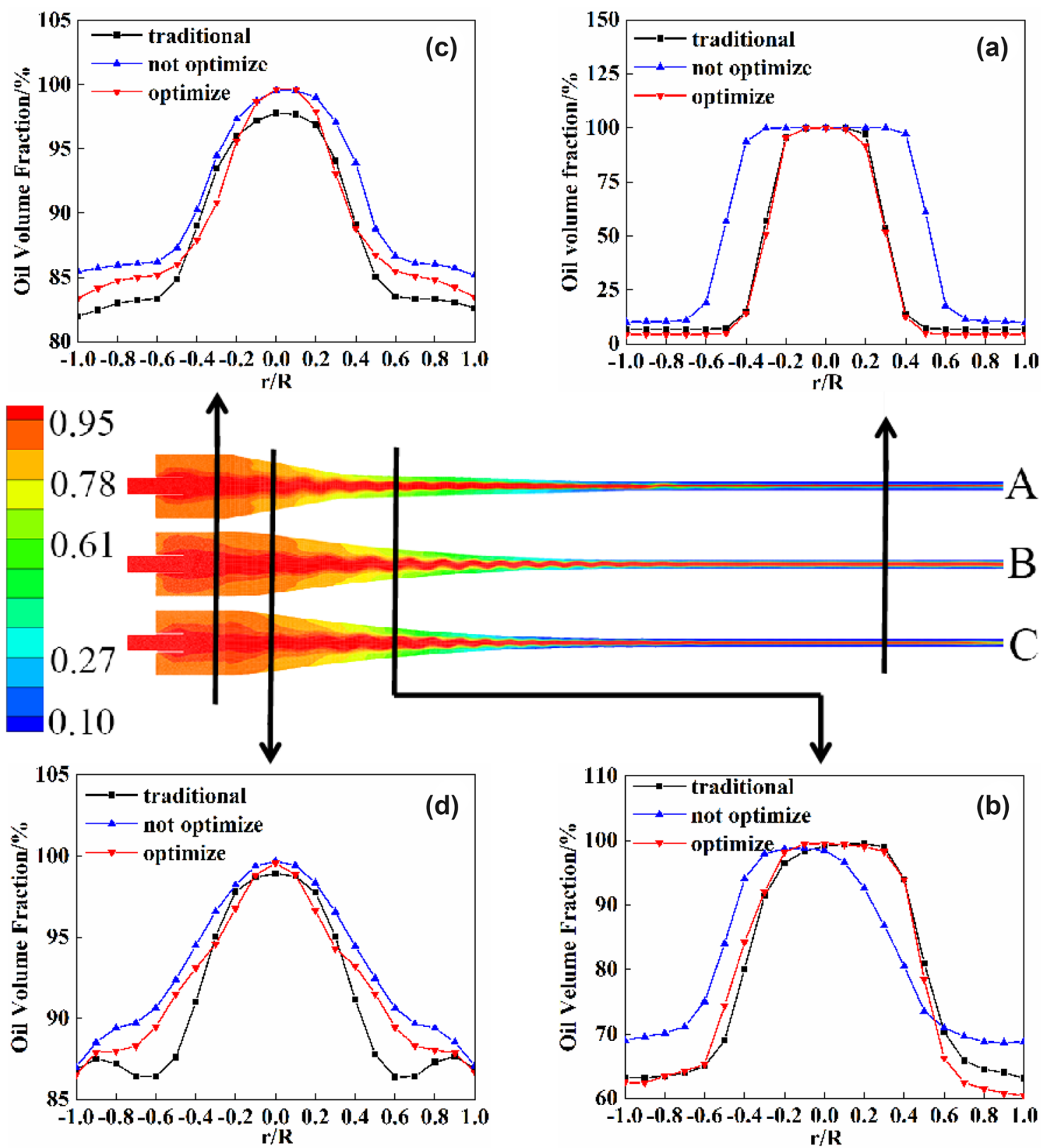

Fig. 12 Contours of oil volume fraction at the section $\mathrm{x}=0 \mathrm{~mm}$ (A: straight double-cone structure, B: not optimized double spherical tangent cone structure, and C: optimized double spherical tangent cone

Fig. 13c, near the underflow port, the droplet size of the nonoptimized double spherical tangent coupling device is larger than that of the straight double-cone coupling device. At the same time, the optimized double spherical tangent coupling device is larger than that of the non-optimized double spherical tangent coupling device, which indicates that the optimized double spherical tangent coupling device is conducive to the discharge of the fluid with higher water content from the underflow port and improves the deoiling rate.

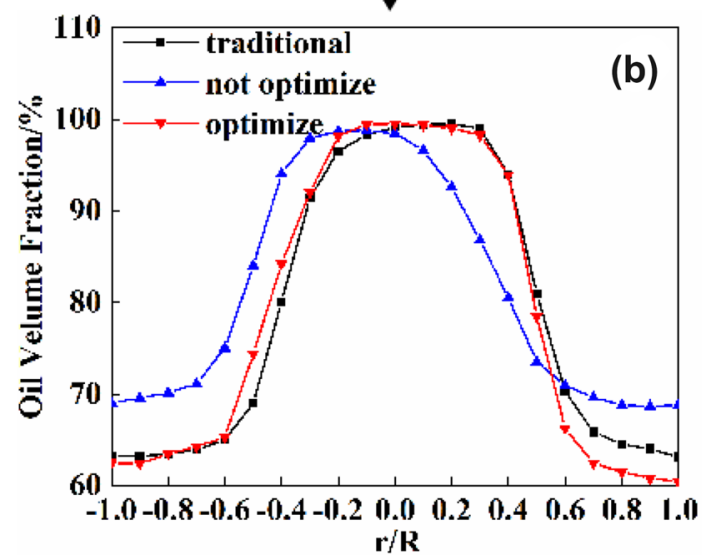

structure) and oil volume distribution of three structures on different sections: a $\mathrm{z}=130 \mathrm{~mm}, \mathbf{b} \mathrm{z}=650 \mathrm{~mm}, \mathbf{c} \mathrm{z}=780 \mathrm{~mm}, \mathbf{d ~ z}=840 \mathrm{~mm}$

Figure 14 shows the droplet diameter distribution of the double-field coupling demulsification-dewatering device with three structures. It can be seen that the droplet diameter distribution of the optimized double spherical tangent coupling device shifts to a larger direction than that of the straight double-cone and non-optimized double spherical tangent coupling device, indicating that the optimized double spherical tangent coupling device is more conducive to the coalescence of droplets, and the droplet is subject 


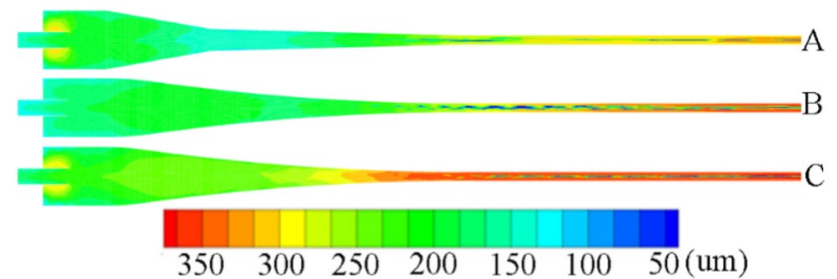

(a)

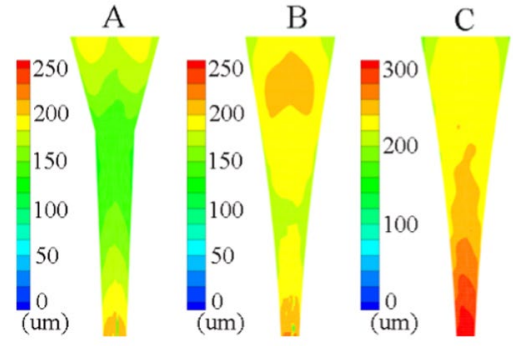

(b)

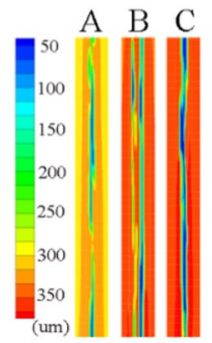

(c)
Fig. 13 a Contours of droplet diameter at the section $x=0 \mathrm{~mm}(\mathrm{~A}$ : straight double-cone structure, B: not optimized double spherical tangent cone structure, and $\mathrm{C}$ : optimized double spherical tangent cone structure); $\mathbf{b}$ at $\mathrm{x}=0$ and $500 \mathrm{~mm}<\mathrm{z}<800 \mathrm{~mm} ; \mathbf{C}$ at $\mathrm{x}=0$ and $0<\mathrm{z}<200 \mathrm{~mm}$

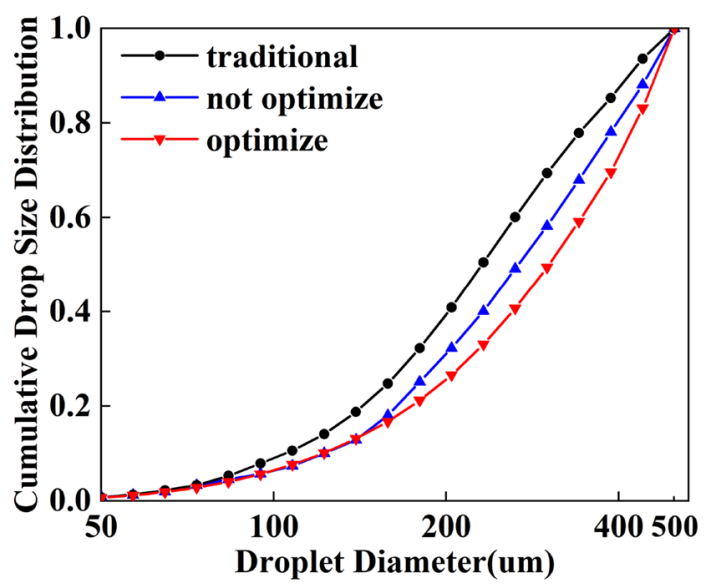

Fig. 14 Droplet diameter distribution under different structures

to greater centripetal force, which promotes the oil-water separation.

\section{Separation efficiency}

Figure 15 shows the separation efficiency under different structures obtained via the numerical method. It can be seen from Fig. 15 that the separation efficiency of the double spherical tangent coupling device is $96.32 \%$, which is $6.13 \%$ and $1.82 \%$ higher than that of the straight double-cone coupling device and the non-optimized double spherical tangent coupling device, respectively, indicating that the optimized

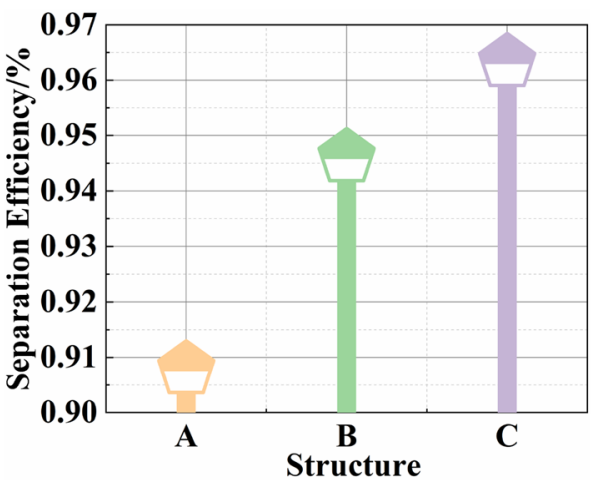

Fig. 15 Separation efficiency under different structures (A straight double-cone structure, $\mathbf{B}$ not optimized double spherical tangent cone structure; $\mathbf{C}$ optimized double spherical tangent cone structure

double spherical tangent coupling device has better separation performance. The reason is that the coupling device carries out a lot of oil-water separation in the double-cone region. Because the straight double-cone coupling device is not smooth in the cone region, the flow field will vibrate in the cone region, which is not conducive to the coalescence of droplets. The double spherical tangent coupling device can solve this problem well. In the cone region, the connection is smooth. Compared with the coupling device with straight double cone, for the device with double spherical tangent cone, the flow field is more stable, and the static pressure value is larger, the pressure drop is smaller, and the energy loss is low. The larger the tangential velocity is, the larger the centrifugal force is, which is conducive to the droplet migration to the wall. The oil volume fraction near the overflow port is larger, which is conducive to the discharge of emulsion with higher oil content from the overflow port. The oil volume fraction on the wall of the underflow port is smaller, which is conducive to the outflow of emulsion with higher water content from the underflow port. The larger droplet size is conducive to improve the separation efficiency of the device.

\section{Conclusion}

In this study, a new double-field coupling device with double spherical tangent cone structure is presented. In the numerical calculation, the droplet coalescence and breakup equations are added to the continuity equation, electric field governing equation, and momentum conservation equation to realize the coupling effect of CFD and PBM. The double spherical tangent cone structure of the coupling device is optimized. The flow field and separation efficiency of the double spherical tangent and the straight double-cone coupling device are analyzed, and confirmatory experiments

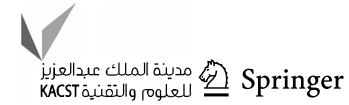


are carried out. The results show that the numerical model can reasonably predict the separation performance of the coupling device. The separation efficiency of the optimized double spherical tangent coupling device is higher than that of the straight double-cone and non-optimized double spherical tangent coupling devices. Under the same conditions, the optimized double spherical tangent coupling device has a lower pressure drop and less energy loss. The tangential velocity on the same section is large, increasing the droplet size, promoting droplet migration to the wall, and enhancing the oil-water separation ability of the emulsion. The oil volume fraction of the optimized double spherical tangent coupling device is higher near the overflow port, and near the underflow, showing that the optimized double spherical tangent coupling device is more conducive to the discharge of fluid with high oil content from the overflow and the outflow of fluid with low oil content from the underflow port, which improves the separation efficiency of the coupling device. The separation efficiency of the optimized double spherical tangent coupling device is $6.13 \%$ and $1.82 \%$ higher than that of the straight double-cone and the non-optimized double spherical tangent coupling device, respectively. The optimized double spherical tangent coupling device has the best separation performance.

Acknowledgements I am grateful to the Chongqing Technology and Business University, Engineering Research Centre for Waste Oil Recovery Technology and Equipment of Ministry of Education and China University of Petroleum (East China) for providing the continuous support during the processing of the present work.

Funding This work was partially supported by grants from the National Natural Science Foundation of China (Grant No. 22178036 and No. 22008016), CSTC projects (Grant No. cstc2019jscx-gksbX0032, cstc2019jcyj-msxmX0296 and cstc2020jcyj-msxmX0157), projects of science and technology research program of Chongqing Education Commission of China (Grant No. KJQN202100817, KJQN201900825, KJZD-K202000803 and KJZD-M201900802).

\section{Declarations}

Conflict of interests The authors declare that they have no known competing financial interests or personal relationships that could have appeared to influence the work reported in this paper.

Open Access This article is licensed under a Creative Commons Attribution 4.0 International License, which permits use, sharing, adaptation, distribution and reproduction in any medium or format, as long as you give appropriate credit to the original author(s) and the source, provide a link to the Creative Commons licence, and indicate if changes were made. The images or other third party material in this article are included in the article's Creative Commons licence, unless indicated otherwise in a credit line to the material. If material is not included in the article's Creative Commons licence and your intended use is not permitted by statutory regulation or exceeds the permitted use, you will need to obtain permission directly from the copyright holder. To view a copy of this licence, visit http://creativecommons.org/licenses/by/4.0/.

\section{References}

Adamski RP, Bethke GK, Kini GC, Shanker SK (2018) Systems and processes for separating emulsified water from a fluid stream: US Patent 10597587

Al-Kayiem HH, Osei H, Hashim FM, Hamza JE (2019) Flow structures and their impact on single and dual inlets hydrocyclone performance for oil-water separation. J Petrol Explor Prod Technol 9(4):2943-2952

Al-Kayiem HH, Hamza JE, Lemmu TA (2020) Performance enhancement of axial concurrent liquid-liquid hydrocyclone separator through optimization of the swirler vane angle. J Petrol Explor Prod Technol 10(5):2957-2967

Almeida-Trasvina F, Smith R, Jobson M (2021) Development of an energy-efficient single mixed refrigerant cycle for small-scale LNG production. Ind Eng Chem Res 60(32):12049-12067

Atten P (1993) Electrocoalescence of water droplets in an insulating liquid. J Electrostat 30(93):259-269

Bailes PJ, Watson M (1992) Electrostatic and centrifugal separation of liquid dispersions. UK Patent A, 2249741

Bijesh K, Paul G, Hamed K, Entele TT, Sharmin H, Alejandro M, Habteyes TG (2018) Robust charge transfer plasmons in metallic particle-film systems. ACS Photonics 10:4022-4029

Cernecky J, Plandorova K (2013) The effect of the introduction of an exit tube on the separation efficiency in a cyclone. Braz J Chem Eng 30(3):627-641

Chen J, Hou J, Li G, Xu C, Zheng B (2015) The Effect of Pressure Parameters of a Novel Dynamic Hydrocyclone on the Separation Efficiency and Split Ratio. Sep Sci Technol 50(6):781-787

Cheng J, Li Q, Yang C, Zhang Y, Mao Z (2018) CFD-PBE simulation of a bubble column in OpenFOAM. Chin J Chem Eng 26(9):1773-1784

Chu K, Chen J, Yu A, Williams RA (2017) Numerical studies of multiphase flow and separation performance of natural medium cyclones for recovering waste coal. Powder Technol 314:532-541

Eow JS, Ghadiri M (2002) Electrostatic enhancement of coalescence of water droplets in oil: a review of the technology. Chem Eng J 85(2-3):357-368

Ghadiri M, Eow JS (2003) Separating components of liquid/liquid emulsion using electrostatic force. UK Patent Application, GB2377397, 15

Ghasemi H, Darjani S, Mazloomi H, Mozaffari S (2020) Preparation of stable multiple emulsions using food-grade emulsifiers: evaluating the effects of emulsifier concentration, W/O phase ratio, and emulsification process. SN Appl Sci 2(12):1-9

Gong HF, Dai F, Yu B (2018) Equipment and experiment for Demulsification and Dewatering by Combining High Voltage Pulsed Electric Field with Swirl Centrifugal Field. Modern Chem Ind 38:178-181

Gong HF, Li WL, Zhang XM, Peng Y, Yu B, Ying MC (2020) Simulation of the coalescence and breakup of water-in-oil emulsion in a separation device strengthened by coupling electric and swirling centrifugal fields. Sep Purif Technol 238:116397

Gu GJ, Liu G, Chen B, Tian M, Wu HY (2015) Research progress of physical demulsification technologies and equipment about W/O emulsions. Chem Ind Eng Progress 34(2):319-324

Hagesaether L, Jakobsen HA, Svendsen HF (2002) A model for turbulent binary breakup of dispersed fluid particles. Chem Eng Sci 57(16):3251-3267

Hamza JE, Al-Kayiem HH, Lemma TA (2020) Experimental investigation of the separation performance of oil/water mixture by compact conical axial hydrocyclone. Thermal Sci Eng Progress 17:100358

Hang Y, Zhong CH, Zhang WD, Xiang SU, Wang XX (2015) Research status and prospects of waste lubricating oil combined technology. Mod Chem Ind 35:19-22 
Howarth WJ (1964) Coalescence of drops in a turbulent flow field. Chem Eng Sci 19(1):33-38

Ansys Incorporation (2013) ANSYS Fluent Users Guide 15.0, ANSYS Inc, Canonsburg

Ansys Incorporation (2015) ANSYS Fluent population balance module manual

Jung KJ, Hwang IJ, Kim YJ (2019) Effect of inner wall configurations on the separation efficiency of hydrocyclone. J Mech Sci Technol 33(11):5277-5283

Kang W, Yin X, Yang HB, Zhao YL, Huang ZT (2018) Demulsification performance, behavior and mechanism of different demulsifiers on the light crude oil emulsions. Colloids Surf, A 545:197-204

Lam SS, Liew RK, Jusoh A, Chong CT, Ani FN, Howard A, Chase C (2016) Progress in waste oil to sustainable energy, with emphasis on pyrolysis techniques. Renew Sustain Energy Rev 53:741-753

Li S (2020) Study on oil-water two-phase flow characteristics of the hydrocyclone under periodic excitation. Chem Eng Res Des 159:215-224

Li W, Shen ZH, Liu C, Li Y, Han X (2015) Numerical simulation analyze on multiphase flow in gravity oil-gas-water separator with different inlet type. China Offshore Platform 30(1):48-52

Liu Y, Wang Z (2016) Research Progress in the Factors Influence the Efficiency of Hydrocyclone Separation. Fluid Machinery 44(02):39-42

Liu J, Zhong L, Ren L, Hao T, Zhou Y (2020) Laboratory evaluation of fluidity of heavy oil emulsions in formation pores medium. ACS Omega 6(1):623-632

Long X, Zhang G, Shen C, Sun G, Wang R, Yin L, Meng Q (2013) Application of rhamnolipid as a novel biodemulsifier for destabilizing waste crude oil. Biores Technol 131:1-5

Lv P, Liu Y, Zhang Y, Sun L, Zou J (2020) Optimization of non-ionic surfactants for removing emulsified oil from gas condensate oil-water emulsion in $\mathrm{N}$ oilfield. J Petrol Explor Prod Technol 10(7):3025-3030

Mhatre S, Vivacqua V, Ghadiri M et al (2015) Electrostatic phase separation: A review. Chem Eng Res Des 96:177-195

Motin A (2015) Theoretical and numerical study of swirling flow separation devices for oil-water mixtures. Dissertations \& Theses Gradworks

Motin A, Bénard A (2017) Design of liquid-liquid separation hydrocyclones using parabolic and hyperbolic swirl chambers for efficiency enhancement. Chem Eng Res Des 122:184-197

Mozaffari S, Tchoukov P, Mozaffari A, Atias J, Czarnecki J, Nazemifard N (2017) Capillary driven flow in nanochannels-Application to heavy oil rheology studies. Colloids Surf, A 513:178-187

Mozaffari S, Li WH, Dixit M, Seifert S, Lee S, Kovarik L, Mpourmpakis G, Karim AM (2019) The role of nanoparticle size and ligand coverage in size focusing of colloidal metal nanoparticles. Nanoscale Advances 1(10):4052-4066

Mozaffari S, Ghasemi H, Tchoukov P, Czarnecki J, Nazemifard N (2021) Lab-on-a-chip systems in asphaltene characterization: a review of recent advances. Energy Fuels 35(11):9080-9101
Noik C, Trapy J (2004) Separation Device and Method Comprising a Tubular Electrocoalescer: US 6702947:B2

Oliveira PJ, Issa RI (2003) Numerical aspects of an algorithm for the Eulerian simulation of two-phase flows. Int J Numer Meth Fluids 43(10-11):1177-1198

Praporgescu G, Mihăilescu S (2011) Study the environmental impact of lubricants used in mechanical systems. Ann Univ Petroşani Mech Eng 13:131-136

Qi C, Yu Z, Fei L, Yang Y, Zeng G (2018) A novel photocatalytic membrane decorated with RGO-Ag-TiO2 for dye degradation and oil-water emulsion separation. J Chem Technol Biotechnol 93(3):761-775

Qian H, Li C, Ping L (2017) A mathematical model for predicting oilwater separation efficiency in a de-oiling hydrocyclone. J Dispersion Sci Technol 38(9):1319-1324

Qiu S, Wang G, Zhou S, Liu Q, Wang L (2020) The downhole hydrocyclone separator for purifying natural gas hydrate: structure design, optimization, and performance. Sep Sci Technol 55(3):564-574

Raya SA, Saaid IM, Ahmed AA, Umar AA (2020) A critical review of development and demulsification mechanisms of crude oil emulsion in the petroleum industry. J Petrol Explor Prod Technol 10(4):1711-1728

Rusche H (2003) Computational fluid dynamics of dispersed two-phase flows at high phase fractions. Imperial College London (University of London)

Schütz S, Gorbach G, Piesche M (2009) Modeling fluid behavior and droplet interactions during liquid-liquid separation in hydrocyclones. Chem Eng Sci 64(18):3935-3952

Tian J, Wang HL, Lv W, Huang Y, Liu Y (2020) Enhancement of pollutants hydrocyclone separation by adjusting back pressure ratio and pressure drop ratio. Sep Purif Technol 240:116604

Vakamalla TR, Kumbhar KS, Gujjula R, Mangadoddy N (2014) Computational and experimental study of the effect of inclination on hydrocyclone performance. Sep Purif Technol 138:104-117

Vashahi F, Rezaei S, Dafsari RA, Lee J (2019) Sensitivity analysis of the vane length and passage width for a radial type swirler employed in a triple swirler configuration. Theor Appl Mech Lett 9(6):363-375

Wang BB, Wang XD, Wang TH, Yan WM (2016) Electro-coalescence of two charged droplets under constant and pulsed DC electric fields. Int J Heat Mass Transf 98:10-16

Xu HL, Liu PK, Yang XH, Zhang YK, Jiang LY (2020) Effect of Composite Curved Conical Section on Separation Performance of Hydrocyclone. Metal Mine 03:184-189

Yang DH, He LM, Luo XM, Zhang HL (2012) Investigation on coalescence characteristics of water droplets in new type electrostatic coalescer. J Chem Engi Chinese Univ 26(2):222-227

Zhang XM, Li WL, Gong HF, Peng Y, Yu B (2020) Simulation on the coalescence and breakup of droplet in double field coupling demulsification and dewatering device. Acta Petrolei Sinica (petrol Process Section) 36(02):357-366 